\title{
CONHECIMENTO DA FAMÍLIA ACERCA DA SAÚDE DAS CRIANÇAS DE 1 A 5 ANOS EM UMA COMUNIDADE RIBEIRINHA: SUBSÍDIOS PARA A ENFERMAGEM PEDIÁTRICA ${ }^{a}$
}

\author{
Family knowledge about 1 to 5 -year-old children in a riverain \\ community: subsidies to pediatric nursing. \\ Conocimientos de la familia sobre la salud de los niños entre \\ 1 y 5 años en una comunidad ribereña: contribuciones a la \\ enfermería pediátrica.
}

Maria Vitória Hoffmann ${ }^{1}$

Isabel Cristina Santos Oliveira ${ }^{2}$

\begin{abstract}
RESUMO
0 estudo tem como objeto o conhecimento da família acerca da saúde das crianças de 1 a 5 anos em uma comunidade ribeirinha. Os objetivos são: descrever as experiências de vida da família; analisar o conhecimento da família e discutir as implicações na saúde das crianças da prática assistencial da enfermagem. Trata-se de um estudo de natureza qualitativa, tipo estudo de caso. Como procedimento metodológico, utilizaram-se formulário e entrevista não diretiva em grupo. Por meio da análise temática, constatou-se que os conhecimentos das relações familiares e sociais são permeados por valores culturais, crenças, costumes e condutas. As condições socioeconômicas e a distância geográfica da comunidade favorecem a articulação entre o saber popular e a oferta de serviços de saúde próximos à comunidade. Conclui-se que familiares da comunidade necessitam de esclarecimentos e orientaç̃oes para os problemas de saúde das crianças por parte dos profissionais de saúde; portanto, torna-se relevante a participação da enfermagem com vistas à promoção da saúde da população infantil.

Palavras-chave: Enfermagem. Criança. Família. Comunidade.
\end{abstract}

Palavras-chave: Enfermagem Neonatal. Prematuro. Enfermagem da Família.

\begin{abstract}
This study aims at analyzing the family knowledge about 1 to 5 year old children's health in a riverain community. The objectives are: describing their life experience; analyzing their knowledge; discussing the implications of this knowledge before the children's health in the nurse practice. It is a qualitative case study. The methods comprise a form to and a nondirective group interview. Based on the thematic analysis, it was found that family knowledge built through family and social relationships is embedded with cultural values, beliefs, customs and conducts as, for example, the use of medicinal plants. The socio-economic condition and the geographic distance favor the relationship between popular knowledge and the health services near the community.lt was concluded that the community family members need the health professionals to provide guidance on how to deal with their children's health problems. In this context, the participation of nursing in guiding is relevant in order to promote children's health.
\end{abstract}

Keywords: Nursing. Children. Family. Community.

\section{Resumen}

El objeto del presente estudio fue sondear el conocimiento que tiene la familia sobre la salud de los niños. Los objetivos fueron: describir la experiencia de vida de la familia; analizar el conocimiento que posee la misma; y discutir las implicaciones que tiene con la salud de los niños en el ámbito de la práctica asistencial de la enfermería. Se trata de un estudio de naturaleza mediante el método de estudio de caso. Como procedimientos metodológicos, fueron aplicados cuestionarios y entrevistas no dirigidas en grupo. Con base en el análisis temático, se comprobó que los conocimientos provenientes de las relaciones familiares y sociales son transmitidos a través de valores culturales, creencias, costumbres y conductas. Las condiciones socio-económicas y la distancia geográfica de la comunidad favorecen el vínculo entre el saber popular y la oferta de servicios de salud cercanos a la comunidad. Se concluyó que los familiares miembros de la comunidad necesitan explicaciones y orientaciones por parte de los profesionales de salud y las enfermeras para poder enfrentar los problemas de salud de los niños. Palabras clave: Enfermería. Niño. Familia. Comunidad.

Palabras-clave: Enfermería neonatal. Prematuro. Enfermería de la familia.

'Mestre em Enfermagem pela Escola de Enfermagem Anna Nery da Universidade Federal do Rio de Janeiro/Minter Faculdades Associadas do Espírito Santo. Professora e Chefe do Departamento de Fundamentos de Enfermagem da Escola Superior de Ciências da Santa Casa de Misericórdia de Vitória. Endereço: Brasil. E-mail: hoffmann_vick@ig.com.br, ${ }^{2}$ Doutora em Enfermagem. Professora Associada do Departamento de Enfermagem MédicoCirúrgica da EEAN/UFRJ. Líder do Grupo de Pesquisa-Saúde da criança/Cenário Hospitalar. Pesquisadora CNPq. Brasil. E-mail: chabucris@ig.com.br 


\section{INTRODUCÃO}

A intenção de estudar a temática voltada para as famílias das crianças de uma comunidade do Estado do Espírito Santo teve origem por volta de 1986, durante o Curso de Graduação em Enfermagem e Obstetrícia da Pontifícia Universidade Católica do Paraná, quando tive a oportunidade de acompanhar as atividades desenvolvidas na Unidade de Saúde da referida universidade, em destaque, 0 atendimento às crianças menores de cinco anos.

Em 2003, fui convidada a trabalhar na Escola de Ciências da Saúde da Santa Casa de Misericórdia de Vitória, com o cargo efetivo de professora auxiliar no Curso de Graduação em Enfermagem, de onde surgiu meu envolvimento com o Projeto de Extensão Universitária "Saúde na Foz do Rio Doce". Este projeto tem como objetivo central desenvolver ações de saúde para realização de atividades que contribuam para a melhoria da qualidade de vida das populações menos favorecidas, e levoume a refletir sobre a Atenção à Saúde da Criança e decidir-me em relação ao tema desse estudo. Atuando em instituições de saúde, deparei-me várias vezes com a concepção sobre a saúde e o cuidado das populações, em várias localidades e regiões do Brasil.

Segundo o Ministério da Saúde ${ }^{1}$, em 2001, a taxa de mortalidade por 100 mil habitantes, para a população com menos de 1 ano de idade, por afecções perinatais na região sudeste foi significativamente maior que as demais causa de mortalidade.

Os óbitos por malformaç̃ões congênitas ocuparam o segundo lugar. Na faixa etária de 1 a 4 anos, as causas externas representaram maior risco tanto para as meninas como para os meninos, e em seguida aparecem as doenças do aparelho respiratório e as doenças infecciosas e parasitárias. Nos casos de morbidade hospitalar no município de Linhares/Espírito Santo, conforme a distribuição percentual das internações por grupo de causas e faixas etárias, constata-se que, de 1 a 4 anos, os grupos de causas correspondem às doenças: infecciosas parasitárias (22,9); respiratórias $(31,0)$; digestivas $(6,2)$, dermatológicas $(0,6)$ e endócrinas, nutricionais e metabólicas $(1,5)^{2}$

Segundo o Fundo das Nações Unidas para a Infância ${ }^{3}$, cerca de 100 mil crianças ainda morrem anualmente no Brasil, a maioria por causas evitáveis.

Na década de 80, estudos buscaram conhecer as famílias brasileiras, desenvolvendo pesquisas com enfoque nas famílias de comunidade pesqueira, para compreender suas práticas em relação à saúde, destacando sempre a preocupação em subsidiar e ressaltar a importância da assistência de enfermagem junto às famílias. No País, a prática do cuidar de famílias continua permeada por incertezas, apesar de a família ter sido sempre considerada como cliente da enfermagem. ${ }^{4}$

Neste sentido, consideram que a área de enfermagem familiar está apenas iniciando, o que justifica a necessidade da utilização de instrumental teórico-metodológico desenvolvido em outras áreas de saber, para uma melhor compreensão da família e atuação do enfermeiro.

A familia é considerada a principal parceria nessa missão e deve ser fortalecida com conhecimentos e habilidades para cuidar de suas crianças, garantindo pleno desenvolvimento nos primeiros 5 anos de vida. 0 Grupo de Assistência, Pesquisa e Educação na Área da Saúde da Família (GAPEFAM) busca, em sua trajetória, a compreensão da família em seu processo de viver e ser saudável, identificando a família como foco central do cuidado. ${ }^{5}$

Os membros da família estão unidos por laços consanguíneos, de adoção, interesse e/ou afetividade. Tem identidade própria, possui e transmite crenças, valores e conhecimentos comuns influenciados por sua cultura e nível socioeconômico. A família tem direitos e responsabilidades, vive em um determinado ambiente em interação com outras pessoas e famílias, em diversos níveis de aproximação, e promove meios para o crescimento, desenvolvimento, saúde e bem-estar dos seus membros. ${ }^{3}$

Na 'família saudável', estabelece-se uma interação positiva caracterizada como uma unidade que se autoestima positivamente, onde os membros tentam se colocar um no lugar do outro e expressam sentimentos de afeto, ideias, crenças, valores e conceitos, possibilitando-os desempenharem seus papéis e conviverem e se percebem mutuamente como família. ${ }^{4}$

Todos os aspectos da vida estão permeados pela cultura, este é um processo dinâmico influenciado por diversos fatores socioeconômicos, políticos, históricos e geográficos. ${ }^{6}$

A família saudável com uma estrutura definida cria condições para reflexões sobre as questões do dia-a-dia e de novas alternativas de procedimentos, propiciando critérios de análise e avaliação das informações sobre as condições de saúde dos seus membros, decidindo o tipo de atitude, como procura um hospital, ou uma benzedeira, ou a utilização de tratamentos caseiros, com chás, banhos, entre outros.

Dessa forma, delimito como objeto de estudo o conhecimento da família acerca da saúde das crianças de 1 a 5 anos em uma comunidade ribeirinha. Os objetivos são : descrever as experiências de vida da família quanto à saúde das crianças; analisar o conhecimento da família acerca da saúde das crianças; e discutir as implicações dos conhecimentos da família na saúde das crianças.

\section{ABORDAGEM TEÓRICO-METODOLÓGICA}

0 referencial teórico está vinculado aos estudos de Elsen et al., estes destacando sempre a preocupação em subsidiar e ressaltar a importância da assistência de enfermagem junto ao grupo familiar. A família cria um espaço que visa o interesse, 0 prazer e o bem-estar de todos os membros, partindo da necessidade de compreensão do processo de identificação de problemas, procurando resolver e/ou prevenir as dificuldades na busca de soluções, com uma visão crítica do que acontece à 
sua volta, garantindo, assim, o crescimento, desenvolvimento e a identidade familiar.

A autora e o grupo GAPEFAM definem família saudável aquela que estabelece uma interação positiva caracterizada por um relacionamento direto, de respeito e liberdade, sem tensões, em que os membros tentam se colocar um no lugar do outro, expressando sentimentos de afeto, ideias, crenças, valores e conceitos, o que os possibilita crescer, desenvolver-se, definirse, ajustar-se e desempenhar seus papéis.

0 estudo é de natureza qualitativa tipo estudo de caso 0 cenário é a área urbana isolada de Povoação, no povoado de Pontal do Ipiranga, no distrito de Regência, no município de Linhares do estado do Espírito Santo. Os sujeitos são 17 membros das famílias divididas em cinco grupos, que convivem com crianças de 1 a 5 anos de idade, residentes no referido cenário. A escolha dos familiares foi aleatória, e a participação, voluntária.

Para proceder com a coleta de dados, foi utilizado um formulário a fim de obter dados referentes ao universo familiar e a técnica de entrevista não diretiva em grupo, baseada no discurso livre dos entrevistados. Os temas que subsidiaram a entrevista não diretiva em grupo foram elaborados com base nas cinco ações básicas do Programa de Assistência Infantil à Criança (PAISC) e Atenção Integrada às Doenças Prevalentes na Infância (AIDPI)e foram os seguintes: pessoa que cuida da criança na família; vacina da criança; amamentação (idade); alimentação diária; e cuidado com a criança com problemas de saúde (febre, diarreia, tosse, problemas de pele, queimaduras, fraturas, entre outros).

A coleta foi realizada após a aprovação e liberação pelo Comitê de Ética em Pesquisa da Escola Superior de Ciências da Santa Casa de Misericórdia de Vitória- EMESCAM em 26/04/ 2006 e autorizado pela Secretária Municipal de Saúde do Município de Linhares em 20/09/2006, cenário do estudo.

Para a obtenção dos dados foi utilizado como recurso um gravador, o que proporcionou os depoimentos na íntegra, gravados em fitas magnéticas. A partir da aceitação dos membros das famílias integrantes do estudo, foi respeitado o previsto na Resolução n 196/96 e apresentado o Termo de Consentimento Livre e Esclarecido.

Foi realizada a validação metodológica com vistas a atender os objetivos do estudo. Constatou-se que os temas foram entendidos, e, após a transcrição, iniciou-se a análise temática com a leitura flutuante dos depoimentos, destacando os temas relevantes; em seguida foram agrupados, visando a estruturação dos tópicos do capítulo de análise.

\section{CONHECIMENTO DA FAMÍLIAACERCA DA SAÚDE DAS CRIANÇAS}

Esse item enfoca os conhecimentos das famílias acerca da saúde das crianças de 1 a 5 anos, descrevendo os saberes e experiências de vida delas no tocante ao uso de plantas medicinais, a conduta familiar diante dos problemas de saúde e os aspectos relacionados à vacinação e alimentação das crianças na comunidade.

\section{Saberes e experiências de vida da família: uso de plantas medicinais}

Os chás de plantas medicinais utilizados pelos familiares para os problemas das crianças relacionados aos casos de febre, gripe, tosse e asma são os de aroeira, folhas de laranja, poejo e favaquinha. Para problemas do sistema digestivo, os familiares usam chá de folha de goiaba para conter episódios de diarreia, e no caso de dor abdominal, utilizam os chás de boldo e de erva cidreira.

As práticas baseadas no saber popular não se encontram apenas na falta de esclarecimento ou de recursos financeiros por parte da população; as crenças e práticas baseadas no saber popular e experiências empíricas são adotadas como recursos destinados para manutenção da saúde ou cura das doenças. $^{8}$

A mãe menciona que a criança apresentou algum problema de saúde, na qual ela observa "febre", "tosse", "dor de barriga", "diarreia", "gripe", entre outras; a mãe faz a avaliação, e o sinal para procurar recursos médicos é a febre. ${ }^{9}$ Se a criança não apresenta febre, a mãe prepara soluções caseiras, como chá, sucos, xaropes, remédios caseiros antidiarreicos, entre outros, e não procura assistência médica.

Outros familiares utilizam o chá de folha de laranja com um comprimido de analgésico; estão acostumados em virtude das ocorrências de outros episódios de febre. Cabe lembrar que o serviço médico e os fármacos utilizados pelos familiares não estão excluídos da medicina tradicional, desde que estejam disponíveis. Um familiar mencionou a consulta médica no posto de saúde da comunidade nos casos de emergências ou em doenças reconhecidas como "doenças de médico"

0 conjunto de conhecimentos, ações e procedimentos da medicina tradicional estão baseados em uma tradição oral, que conjuga o "ver fazer" com o "ouvir dizer", que muitas vezes são embasadas em conhecimentos, normas e tabus transmitidos geralmente por um sistema de tutoria de linhagem familiar. ${ }^{10}$

As plantas medicinais também são usadas no preparo de xarope como remédio caseiro, principalmente para as doenças respiratórias, como tosse, sibilo, gripe, bronquite e asma. 0 uso dos remédios naturais ${ }^{6}$ pelos familiares da comunidade foi relato pelos depoentes nas diferentes situações de problemas de saúde, e a prática popular é priorizada sempre que ocorre a possibilidade de solução. Os conhecimentos sobre plantas e remédios populares são adquiridos por experiências vivenciadas anteriormente ou resgatadas de pessoas com referência na comunidade, como as pessoas mais velhas, parentes, familiares, amigos ou até mesmo benzedeiros e curandeiros. Desta forma, o cuidado no campo da saúde é uma busca de interseção entre o mundo do saber popular e uma sabedoria científica.

Os familiares mencionaram o uso de xarope, prescrito pelo médico para a gripe, tosse e bronquite asmática. A família, com suas experiências de vida, têm construído a sua prática de cuidado, identificando o tipo de problema e tomando decisões 
sobre o encaminhamento, ao posto ou ao hospital, ou a utilização de tratamentos caseiros como o uso de chás e xaropes. Este conhecimento popular traz contribuições para 0 entendimento na relação entre a família, a crença, a cultura e as condições sociais de vida dessas pessoas. ${ }^{5}$

Cabe acrescentar que entre os vários recursos que constituem o cuidado à saúde, a crença em Deus está presente e é destacada como condição primordial para que ocorra um resultado favorável, sendo necessária em todas as condutas e procedimentos realizados no tratamento da doença.

Em relação ao uso de banhos preparados com plantas medicinais, são utilizados a aroeira, a folha de laranja e o capim cidreira, para aliviar sintomas como febre, dor e problemas de pele. Por outro lado, outros familiares optaram pelo tratamento médico, em vez dos banhos utilizados na comunidade.

A família volta-se internamente para atender as necessidades individuais de seus membros e desenvolve um verdadeiro processo de cuidar, observando, identificando o problema, definindo a situação e tomando decisão sobre a melhor forma de conduzir o tratamento daquele que necessita de cuidados.

A saúde da família e de seus membros está relacionada não só com os recursos materiais, mas com todas as pessoas que estão à sua volta, inclusive a comunidade e os serviços de saúde e seus profissionais. ${ }^{4}$

\section{Conduta das famílias diante dos problemas de saúde: uma decisão peculiar}

Destacados os diferentes problemas de saúde, sintomatologia e local do atendimento, a conduta familiar nos casos de pneumonia, dor de ouvido e febre, segundo a prescrição médica, foi a utilização dos medicamentos, como os comprimidos.

As autoras ressaltam que a família não é um recipiente passível do cuidado profissional, mas agente do seu próprio processo de viver. ${ }^{4} \mathrm{~A}$ faixa etária tem grande importância na presença da doença respiratória, sendo as crianças mais novas as mais atingidas, em vários contextos socioeconômicos. As frequências encontradas vão de quatro a oito episódios anuais de doenças respiratórias por criança menores de cinco anos. ${ }^{11}$

Compreende-se que essas famílias percebem sinais de certas doenças que acometem seus filhos e têm noções sobre hidratação, alimentação e algumas medicações antitérmicas. 0 autor também coloca 0 uso dos remédios pelas mães, enfatizando que elas possuem remédios conhecidos, compondo uma farmácia familiar. A medicina familiar é considerada imitativa, ou seja, as mães reproduzem gestos e palavras dos médicos, memorizando alguns medicamentos, sintomas e nomes de doenças, valorizando a terapêutica medicamentosa. ${ }^{11}$

Um familiar menciona a prática popular referente à hérnia de umbigo, explicando como ocorre a extração. 0 referido comportamento pode ser justificado quando a autora descreve $\mathrm{e}^{12}$ que o mundo imaginal "é todo este mundo de significados, de ideias, de fantasias, de evocação de figuras já percebidas e não percebidas, de crenças, de valores,... onde o ser humano está mergulhado"

O familiar, de acordo com a sua realidade, elabora conceitos baseados no seu entendimento, estilo de vida, condição social e conceitos preexistentes da prática diária, experiência de vida e significados. ${ }^{12}$ A unidade de saúde e o hospital foram citados por quatro familiares diante dos problemas de saúde caracterizados como acidente doméstico: corte com caco de vidro; batida na porta do carro; fratura por queda de nível e queimadura.

Um dos aspectos mais difíceis na prática com famílias é a questão de que nem sempre a comunicação é efetiva, pelo fato de a interação profissional, geralmente, estar baseada nos valores do profissional, em contraste com a visão de mundo da família, o que gera problemas, levando a julgamentos sobre o comportamento da família. ${ }^{4}$

No Brasil, a prevalência de injúrias envolvendo crianças em ambiente doméstico é apenas estimada, uma vez que não há estudos que identifiquem esse cenário de forma segura. 0 domicílio é o ambiente no qual as crianças entre 1 e 5 anos de idade ficam mais tempo, têm maior liberdade para se locomover e se interessam mais pelo que existe ao redor. Elas necessitam de proteção passiva, que consiste em ter um ambiente protegido. $^{13}$

Os acidentes domésticos mais frequentes, segundo a faixa etária de 0 a 1 ano, são asfixias, sufocações, quedas, queimaduras, corpos estranhos, intoxicações, ferimentos variados e, na faixa etária de 2 a 5 anos, são quedas, asfixias, sufocação, queimaduras, afogamentos, intoxicações, choque elétrico, atropelamento, traumatismo. ${ }^{14}$

Os traumatismos de partes moles podem ser classificados de diversas formas como: contusões (edema, hematomas, lesão sem continuidade) e ferimentos (lesão com solução de continuidade da pele, músculos e tendões). As classificações das lesões são: abrasivas - feridas rasas; incisas - feridas profundas; cortantes - feridas profundas de faca, machado e caco de vidro; perfurantes - feridas profundas, que seriam causadas por ponta de tesoura, ou prego; e lacerantes - traumas, quedas e mordeduras de animais. ${ }^{14}$

A queimadura é um dos acidentes mais frequentes em pediatria, ocupando o primeiro lugar dentro da ocorrência domiciliar. As lesões produzidas por líquidos quentes são as mais comuns, especialmente em menores de 5 anos, enquanto a maioria dos acidentes fatais é determinada pela chama. ${ }^{13}$

Um familiar menciona sua conduta no caso de queimaduras da mão de seu filho e que este aprendeu a não chegar perto do forno quente. A dificuldade reside basicamente nos conflitos de valores. Nesse processo, cada qual entra com uma possibilidade que the é peculiar, e que consiste em um certo conhecimento que as pessoas utilizam para definir a experiência e agir conforme a interpretação. ${ }^{14}$ 
Os profissionais de saúde necessitam repensar a relação da família com o serviço de saúde, buscando reconhecer as opiniões trazidas pela população, além de se envolverem com a realidade de saúde e de vida da população. ${ }^{11}$

\section{A família diante da imunização das crianças: uma conduta relevante}

Constata-se que a imunização das crianças da comunidade é uma conduta adotada pelos familiares, ressaltando o cartão da criança. Pode-se supor que a imunização preconizada pelo Ministério da Saúde está ao alcance da comunidade, cenário do estudo.

A família, principalmente a mulher, é responsável pelo cuidado relacionado à proteção das doenças. Em qualquer tipo de família tem-se a expectativa de que ela ofereça alimentação e 0 aleitamento materno, educação, cuidados e proteção, promovendo melhor condição de vida para seus membros. ${ }^{16}$

0 esquema básico de vacinação da criança é importante e os pais devem ser informados sobre o papel das vacinas na saúde de seus filhos, bem como a importância das anotações feitas sobre as vacinas administradas e 0 agendamento das próximas no cartão da criança, conforme as linhas de Cuidado da Atenção Integral da Saúde da Criança e Redução da Mortalidade Infantil do Ministério da Saúde.

Dois familiares mencionaram possível reação após a criança receber a vacina. As reações mais frequentes às vacinas são de natureza benigna, incluindo febre, mal-estar, reação no local da aplicação (dor, edema, hiperemia, calor, pequena lesão seguida de cicatriz) ou exantema discreto, via de regra, sem exigir cuidados especiais. As doenças preveníveis pela imunização, como poliomielite, hepatite $B$, sarampo, rubéola, caxumba, tétano, coqueluche, difteria, entre outras, devem ser notificadas imediatamente pela equipe de saúde ${ }^{15}$.

Um familiar mencionou a unidade básica de saúde como o local onde as crianças são vacinadas: a imunização é considerada uma atividade quase que exclusiva do serviço público de saúde. A unidade de saúde deve garantir o funcionamento contínuo da sala de vacinação, sem restrições de horários, para não perder a oportunidade de imunizar as crianças. As equipes de saúde devem fazer busca ativa aos faltosos e realizar o controle do cartão de vacinação; constato que as famílias revelam um conhecimento a respeito da vacinação efetuada nas unidades básicas de saúde e periodicamente promovida pelas campanhas.

Outro aspecto abordado por um familiar é a importância da vacina para a criança; uma ${ }^{17}$ proporção variável de menores de 5 anos não passa pelo esquema completo de vacinação para sua idade, e muitas outras crianças, embora com esquema completo, em vez de receber as doses de vacina nas datas mais apropriadas, recebem-nas com atraso, aumentando o risco de adoecer.

Deste modo, ressalta-se a importância da participação da família no cuidado da criança, visto que representa segurança e afeto para ela. ${ }^{4}$

\section{Alimentação das crianças na comunidade ribeirinha: hábitos da família}

Constata-se que existe o hábito de aleitamento materno até os seis meses de idade ou mais. Supõe-se que as famílias da comunidade cenário do estudo têm conhecimento sobre a importância do aleitamento materno nos primeiros seis meses de vida. Vale acrescentar que, de acordo com a caracterização dos sujeitos do estudo, o aleitamento materno exclusivo foi maior nas mulheres mais instruídas.

A Organização Mundial da Saúde (OMS), o Fundo das Nações Unidas para a Infância (UNICEF) ${ }^{3}$ e o Ministério da Saúde (MS) 7 propõem que o aleitamento materno exclusivo é quando a criança recebe somente o leite materno, diretamente da mama ou extraído, não aceita nenhum outro líquido ou sólido, com exceção de gotas ou xaropes de vitaminas, minerais ou medicamentos.

0 aleitamento materno exclusivo é considerado um dos pilares fundamentais contra doenças e para a promoção de afeto, sendo recomendado por quatro a seis meses de vida da criança. $^{18}$

A necessidade do aleitamento artificial, quando a mãe tema necessidade de trabalhar, demonstra a transformação ocorrida na estrutura familiar diante das dificuldades socioeconômicas. 0 desmame precoce ocorre porque as ações de amparo à mulher trabalhadora que está amamentando não são cumpridas, conforme Constituição Federal de 1988 e Consolidação das Leis do Trabalho (CLT), as quais preveem o direito da mulher de trabalhar uma hora a menos para amamentar a criança até completar seis meses de idade. ${ }^{7}$

Outra situação descrita pelo familiar é a conduta médica para o desmame. A alimentação complementar é o conjunto de outros alimentos além do leite materno, inclusive alimentos inadequados à saúde da criança, como: chás, água açucarada, leite em pó erroneamente diluído, sopas diluídas, entre outros. ${ }^{18}$

Constata-se que as famílias ofertam pouca quantidade de frutas, verduras e vegetais folhosos às crianças, muito embora haja grande variedade desses alimentos ricos em minerais e vitaminas que podem ser adquiridos na horta comunitária da comunidade local de Povoação.

A partir dos seis meses, o uso exclusivo de leite materno não supre todas as necessidades nutricionais da criança, sendo necessária a introdução de alimentos complementares. Também nessa idade, a maioria das crianças atinge o estágio de desenvolvimento geral e neurológico (mastigação, deglutição, digestão e excreção) que as habilita a receber outros

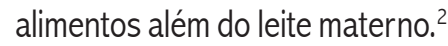

Segundo o MS, as refeições de sal devem ser semelhantes às do adulto, e podem ser consumidos todos os tipos de carnes e vísceras. Deve-se estimular o consumo de frutas e verduras, lembrando que aquelas de folhas verde-escuras apresentam maior teor de ferro, cálcio e vitaminas. Deve-se evitar a utilização de alimentos artificiais e corantes. ${ }^{17}$ 
A relação de apoio familiar nas famílias saudáveis se intensifica principalmente nos momentos em que ocorrem mudanças na sua vida cotidiana. ${ }^{4} 0$ modo de fazer a alimentação para as crianças foi mencionado por dois familiares; ainda, um familiar descreve o cuidado com os alimentos.

Muitos alimentos regionais de alto valor nutritivo, disponíveis e utilizados na alimentação da família, não são dados às crianças nos primeiros anos de vida em decorrência das crenças e tabus do tipo de alimentos, remoso, frio, quente, fraco, entre outros.

Quanto às recomendações médicas em relação ao período de introdução da alimentação complementar, deve-se atentar para o elevado risco para a criança da oferta de alimentos inadequados e risco de contaminação devido à manipulação e preparo inadequados, favorecendo a ocorrência de doenças diarreicas e desnutrição. A orientação para as mães é de fundamental importância para que a alimentação contenha os seguintes grupos alimentares: cereal ou tubérculo, alimento proteico de origem animal, leguminosas e hortaliças; devem ser evitados alimentos industrializados (refrigerantes, café, chá, embutidos, dentre outros). ${ }^{18}$

\section{CONSIDERACÕES FINAIS}

Este estudo permitiu entender o conhecimento da família acerca da saúde das crianças de 1 a 5 anos em uma comunidade ribeirinha

A abordagem qualitativa, tipo estudo de caso e a entrevista não diretiva em grupo propiciaram a interação da autora desse estudo com os sujeitos da pesquisa a fim de obter 0 conhecimento das famílias em um contexto específico.

Autilização de plantas medicinais para diferentes problemas de saúde das crianças, destacando as indicações e o modo de preparo, é experiência vivenciada pelos familiares, permeada pelo saber popular transmitido pelas gerações anteriores. Algumas mães que procuraram o médico ou a unidade de saúde foram as pessoas com maior grau de instrução.

Por outro lado, quando o familiar não encontrava o médico, muitas vezes, procura as pessoas da comunidade com os conhecimentos passados de geração em geração, da estirpe familiar, de vizinhos, ou de amigos. Alguns familiares procuravam os "curandeiros", reforçando a credibilidade que 0 saber popular tem para a comunidade cenário de estudo.

A imunização das crianças de 1 a 5 anos é uma conduta valorizada pelos familiares da comunidade, ressaltando as reações vacinais e as condutas adotadas.

Quanto à alimentação predominante no primeiro ano de vida da criança, destaca-se 0 aleitamento materno e a utilização de outros tipos de alimentação láctea. Entretanto, a alimentação das crianças após os seis meses torna-se deficiente em vitaminas e minerais.

Evidencia-se que os conhecimentos dos familiares construídos através das relações familiares e sociais são permeados por valores culturais, crenças, costumes e condutas, como, por exemplo, a utilização de plantas medicinais.
As condições socioeconômicas e a distância geográfica da comunidade favorecem a articulação entre o saber popular e a oferta de serviço de saúde mais próxima do município. A familia não pode ser vista apenas como aquela que deve cumprir as determinações da equipe de saúde, considerando que ela assume a responsabilidade pela saúde dos seus membros, e sua participação deve ser incentivada visando à obtenção de informações, dúvidas e dificuldades na promoção da saúde.

0 estudo constatou a importância e a necessidade de a prática de enfermagem estar voltada às famílias que cuidam das crianças na comunidade, buscando observar, escutar, aprender e entender os familiares no seu contexto socioeconômico e cultural. Assim, essa prática deve estabelecer metas direcionadas à prevenção e educação à saúde com vistas a minimizar os problemas de saúde mais comuns das crianças, em destaque, de 1 a 5 anos de idade.

Considerando que o enfermeiro deve saber sobre os conhecimentos da família para atuar em diferentes cenários, no caso em comunidades ribeirinhas, acreditam que a minha experiência como professora inserida no Projeto Comboios da Saúde, que faz parte do Projeto de Extensão "Saúde na Foz do Rio Doce", e também no desenvolvimento dessa dissertação de mestrado, foi fundamental para o meu aprimoramento profissional e pessoal.

\section{REFERÊNCIAS}

1.Ministério da Saúde (BR). Secretaria de Vigilância em Saúde. Departamento de Análise de Situação de Saúde. Saúde Brasil 2004: uma análise da situação de saúde. Brasília (DF); 2004.

2.Ministério da Saúde (BR). Indicadores municipais de saúde: morbidade hospitalar: município de Linhares/ES. [on-line] [citado 13 out 2006] Disponível em: http://portal.saude.gov.br/portal/ aplicacoes/tabfusion/tabfusion.cfm.

3.Fundo das Nações Unidas para a Infância -UNICEF. Prioridades do UNICEF para 2006. [on-line] [citado 07 set 2006]. Disponível em: http://www.unicef.org/brazil/prioridades06.htm

4.Elsen I, organizadora. Marcos para a prática de enfermagem com famílias. Florianópolis (SC): Ed. UFSC; 1994.

5.Althoff CR, Elsen I, Laurindo AC. Família: o foco de cuidado na enfermagem. Texto \& Contexto Enferm 1998 maio/ago;7(2): 32027.

6.Budó MLD, Resta DG, Denardin JM, Ressel LB, Borges ZN. Práticas de cuidados em relação à cultura e as alternativas populares. Esc Anna Nery Rev Enferm 2008 mar;12(1): 90-6.

7. Ministério da Saúde (BR). Centro de Documentação. Assistência integral à saúde da criança: ações básicas. Brasília (DF); 1984.

8.Barbosa MA, Siqueira KM, Brasil V, Bezerra ALQ. Crenças populares e recursos alternativos como práticas de saúde. Rev Enferm UERJ 2004 jan/abr;12(1): 38-43.

9.Mello DF, Ferriani MGC. Estudo exploratório de opiniões de mães sobre a saúde das crianças menores de 05 anos. Rev Latino-am Enfermagem 1996 jul. 4(2): 87-100. 
10 Negri Filho AA, Kummer SC. A medicina tradicional e as práticas alternativas de saúde. In: Ducan BB, Schmidt MI, Giugliani ERJ, organizadores. Medicina ambulatorial: condutas clínicas em atenção primária. $2^{\mathrm{a}}$ ed. Porto Alegre (RS): Artes Médicas Sul; 1996.

11.Monteiro Al, Ferriani MGC. Atenção à saúde da criança: perspectiva da prática de enfermagem comunitária. Rev Latino-am Enfermagem 2000 jan; 8(1): 99-106.

12.Nitschke RG, Morais EP, Pfeiffer AS, Elsen I. Família saudável: um estudo sobre o conceito e sua aplicabilidade na assistência. Texto \& Contexto Enferm 1992 jul/dez; 2(1): 152-66.

13.Paes CEN, Maciel W. Injúrias não-intencionais (acidentes): riscos no ambiente doméstico. In: Lopez FA, Campos Junior D, organizadores. Tratado de pediatria. São Paulo (SP): Manole; 2007. p.65-6.
14.Waksman H. Injúrias não-intencionais (acidentes): ferimentos de partes moles. In: Lopez FA, Campos Junior D, organizadores. Tratado de pediatria. São Paulo(SP): Manole; 2007. p. 78-9.

15.Grisi SJFE, coordenador. Estratégia atenção integrada às doenças prevalentes da infância (AIDIP). Washington, DC (USA): OPAS; 2005.

16.Kummer SC, et al. Evolução do padrão de aleitamento materno. Rev Saude Publica. 2000 abr; 34(2): 143-48.

17.Sociedade Brasileira de Pediatria. Manual de orientação: alimentação do lactente, alimentação do pré-escolar, alimentação do escolar, alimentação do adolescente, alimentação na escola. São Paulo (SP): SBP; 2006.

18.Ministério da Saúde (BR). Guia alimentar para crianças menores de 2 anos. Brasília (DF): OPAS; 2002.

\section{NOTA}

${ }^{a}$ Este texto constitui uma síntese da dissertação de mestrado em enfermagem do Programa de Pós-Graduação em Enfermagem da Escola Enfermagem Anna Nery da Universidade Federal do Rio de Janeiro e o Minter/Faculdades Associadas do Espírito Santo(FAESA)Defesa em 29 de junho de 2007. 